\title{
Modeling of Hydrogen Diffusion Behavior Considering the Microstructure of Duplex Stainless Steel Weld Metal ${ }^{*}$
}

\author{
by Yuhei Shibamoto**, Yoshiki Mikami*** and Masahito Mochizuki***
}

\begin{abstract}
Hydrogen induced cracking is usually evaluated by totally charged hydrogen concentration and macroscopically applied stress. However, microscopic distributions of stress and hydrogen concentration should be formed at the scale of microstructure. The stress concentration and hydrogen accumulation would be remarkable in duplex stainless steels and its welds, because they usually consist of ferritic and austenitic phases with different strength and diffusion properties. In this study, the effect of microstructure in duplex stainless steel on hydrogen diffusion behavior was investigated as a first step toward the evaluation of hydrogen induced fracture at microscopic scale. A series of finite element simulations have been performed under microstructure models considering duplex stainless steels and its weld metals. The hydrogen diffusion behavior was strongly influenced by the continuity of austenite phases which have lower diffusion constant compared to ferrite phases. The diffusible hydrogen accumulated to the location where stress concentration occurred due to microscopically different mechanical properties, however, the effect of stress concentration at microscopic scale was not significant for the overall diffusion behavior when the stress gradient at macroscopic scale was relatively small.
\end{abstract}

Key Words: $\quad$ Hydrogen Diffusion, Duplex Stainless Steel, Microstructure, Finite Element Simulation, Weld Metal

\section{Introduction}

Duplex stainless steels are used in marine and offshore structures due to their balanced mechanical strength and corrosion resistance ${ }^{1,2)}$. However, there is a possibility of the occurrence of hydrogen-induced cracking ${ }^{3)}$. The environments in which the duplex stainless steels are used are usually corrosive and are therefore susceptible to hydrogen-induced cracking. Currently, hydrogen-induced cracking is evaluated on the basis of totally charged hydrogen concentration and macroscopically applied stress ${ }^{4,5)}$. However, it is expected that microscopic distributions of stress and hydrogen concentration occur at the microstructure level in duplex stainless steels because they consist of ferritic and austenitic phases with different strengths and diffusion properties. Therefore, the stress concentration and hydrogen accumulation would be remarkable in duplex stainless steels and its welds at the microscopic scale. The inhomogeneous distribution of hydrogen should be taken into consideration in the evaluation of hydrogen-induced cracking of dual phase materials.

In this study, the effect of microstructures in duplex stainless steel on the hydrogen diffusion behavior is discussed using finite element analysis as a first step towards the evaluation of hydrogen-induced fractures at microscopic scales.

\footnotetext{
*Received: 2016.10.17

${ }^{* *}$ Non Member, Graduate School of Engineering, Osaka University

${ }^{* * *}$ Member, Graduate School of Engineering, Osaka University
}

\section{Numerical Simulation Method}

\subsection{Numerical formulation of hydrogen diffusion}

Hydrogen diffusion was numerically simulated based on the following formulation ${ }^{6}$ ) by the finite element method. Mass diffusion is defined by the law of conservation of mass described by Eq. (1):

$$
\int_{V} \frac{d c}{d t} d V+\int_{S} \boldsymbol{n} \cdot \boldsymbol{J} d S=0
$$

where $V$ is the arbitrary volume, $c$ is the concentration, $t$ is the time, $S$ is the surface of $V, \boldsymbol{n}$ is the outward normal vector of $S$, and $\boldsymbol{J}$ is the concentration flux.

Assuming that the driving force of mass diffusion is the gradient of the generalized chemical potential $\mu$, the concentration flux $\boldsymbol{J}$ is described by Eq. (2):

$$
\boldsymbol{J}=-\frac{\boldsymbol{D} c}{R T} \frac{\partial \mu}{\partial x}
$$

where $\boldsymbol{D}$ is the diffusion coefficient, $R$ is the gas constant, $T$ is the temperature, and $x$ is the position vector. The generalized chemical potential is defined by Eq. (3):

$$
\mu=\mu^{0}+R T \ln \varphi+p \overline{V_{H}}
$$

where $\mu^{0}$ is the standard chemical potential, $\varphi$ is the normalized concentration ( $=c / s$, where $s$ is the solubility), $p$ is the hydrostatic pressure, and $\overline{V_{H}}$ is the partial molar volume of hydrogen in the solid solution. Equations (2) and (3) yield Eq. (4):

$$
\boldsymbol{J}=-s \boldsymbol{D}\left(\frac{\partial \varphi}{\partial x}+\varphi \ln \varphi \frac{\partial \ln T}{\partial x}+\frac{\varphi \overline{V_{H}}}{R T} \frac{\partial p}{\partial x}\right)
$$


In this study, the temperature was constant; therefore, Eq. (4) is expressed by Eq. (5):

$$
\boldsymbol{J}=-s \boldsymbol{D}\left(\frac{\partial \varphi}{\partial x}+\frac{\varphi \overline{V_{H}}}{R T} \frac{\partial p}{\partial x}\right)
$$

The terms on the right side of Eq. (5) indicate that the gradients of concentration and hydrostatic pressure are the driving force for the hydrogen diffusion. The simulation in this study was performed by using the finite element analysis software Abaqus, which has the ability to simulate hydrogen diffusion based on the above formulations.

\subsection{Model description}

The dimensions and boundary conditions of the model used in hydrogen diffusion analysis are shown in Fig. 1. The model was composed of $70 \%$ of ferrite phase and $30 \%$ of austenite phase. The initial hydrogen concentration was set to $0 \mathrm{ppm}$ and the temperature was $20^{\circ} \mathrm{C}$ throughout the simulation. A constant hydrogen concentration of $0.033 \mathrm{ppm}$ was applied to Edge-A of the model, and $0 \mathrm{ppm}$ to Edge-B. This boundary conditions model that the diffusible hydrogen is charged from Edge- $\mathrm{A}$ and is discharged from Edge-B. Both sides of the model were insulated. The direction of hydrogen diffusion was the positive $y$-direction. The evolution of the averaged hydrogen concentration on the evaluation line located in the middle of the model in $y$ direction was mainly discussed.

In order to discuss the influence of the microscopic stress distribution on the hydrogen concentration distribution and diffusion behavior, a stress analysis was performed under plane-strain condition before hydrogen diffusion analysis. The model used in the stress analysis is shown in Fig. 2. The dimensions and composition of this model was same as those of the model used in the hydrogen diffusion analysis. In the stress analysis, the displacement of Edge-C was fixed, and $1 \%$ tensile strain in $x$-direction was applied by forced displacement on Edge-D. Hydrostatic pressure was calculated by the stress analysis and was introduced to hydrogen diffusion analysis as the hydrostatic pressure stress term in Eq. (5).

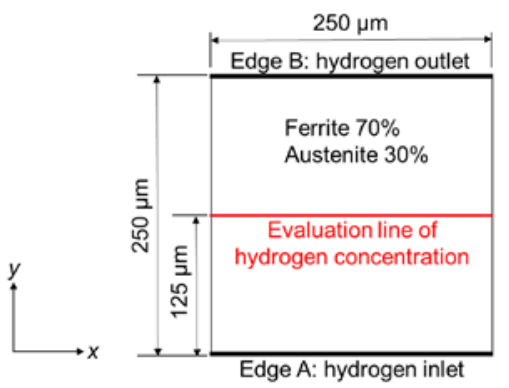

Fig. 1 Dimension and boundary conditions of the numerical simulation model used in hydrogen diffusion analysis.

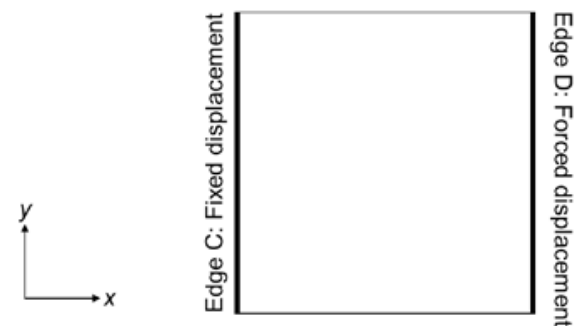

Fig. 2 Boundary conditions of the numerical simulation model used in stress analysis.

In order to investigate the effect of microstructure on hydrogen diffusion, the microstructures of the numerical simulation models were varied as shown in Fig. 3. Keeping the fraction of the austenite phase (black in Fig. 3) at $30 \%$, five different microstructures were prepared. In the microstructures WM-1, WM-2, and WM-3, the austenite phase that had precipitated in ferrite grain boundaries were modeled. The models WM-1, WM-2, and WM-3 are the models for duplex stainless steel weld metals. In WM-1, the ferrite grain boundary was completely covered by the austenite phase, therefore, the austenite phase was continuous. In models WM-2 and WM-3, the austenite phases in the ferrite grain boundary decreased and those in the grain interiors increased keeping the austenite phase fraction. Consequently, the continuity of the austenite phase decreased. The values of the grain boundary coverage by austenite phase in models WM-1, WM-2, and WM-3 were $1,0.9$, and 0.8 , respectively. The grain boundary coverage by austenite phase is defined as the ratio of the grain boundary length covered by the austenite phase to the total grain boundary length. In the microstructure BM-1, ferrite and austenite phases were randomly distributed and the aspect ratio of austenite phase was 1 . In the microstructure BM-2, layered austenite phases were distributed in the ferrite matrix and the aspect ratio of austenite phase was 4 . Material properties such as mechanical properties and diffusion constants used in the numerical simulation are summarized in Table 1. In this study, the effect of the plastic strain on diffusion properties was not taken into consideration.

Table 1 Mechanical properties, diffusion coefficient and solubility ${ }^{3,7,8)}$.

\begin{tabular}{|c|c|c|}
\hline Properties & Ferrite & Austenite \\
\hline Young's modulus, $E(\mathrm{MPa})$ & 200000 & 193000 \\
\hline Poisson's ration, $v$ & 0.3 & 0.3 \\
\hline Yield strength, $\sigma \mathrm{Y}(\mathrm{MPa})$ & 305 & 255 \\
\hline Tensile strength, $\sigma_{\mathrm{T}}(\mathrm{MPa})$ & 550 & 590 \\
\hline Diffusion coefficient, $\boldsymbol{D}\left(\mathrm{m}^{2} / \mathrm{s}\right)$ & $6.0 \times 10^{-113)}$ & $1.4 \times 10^{-167)}$ \\
\hline Solubility, $s\left(\mathrm{ppm} \cdot \mathrm{mm} / \mathrm{N}^{1 / 2}\right)$ & $0.033^{3)}$ & $32.51^{3)}$ \\
\hline $\begin{array}{c}\text { Partial molar volume, } \\
\overline{V_{H}}\left(\mathrm{~mm}^{3} / \mathrm{mol}\right)\end{array}$ & $2000^{8)}$ & $2000^{8)}$ \\
\hline
\end{tabular}



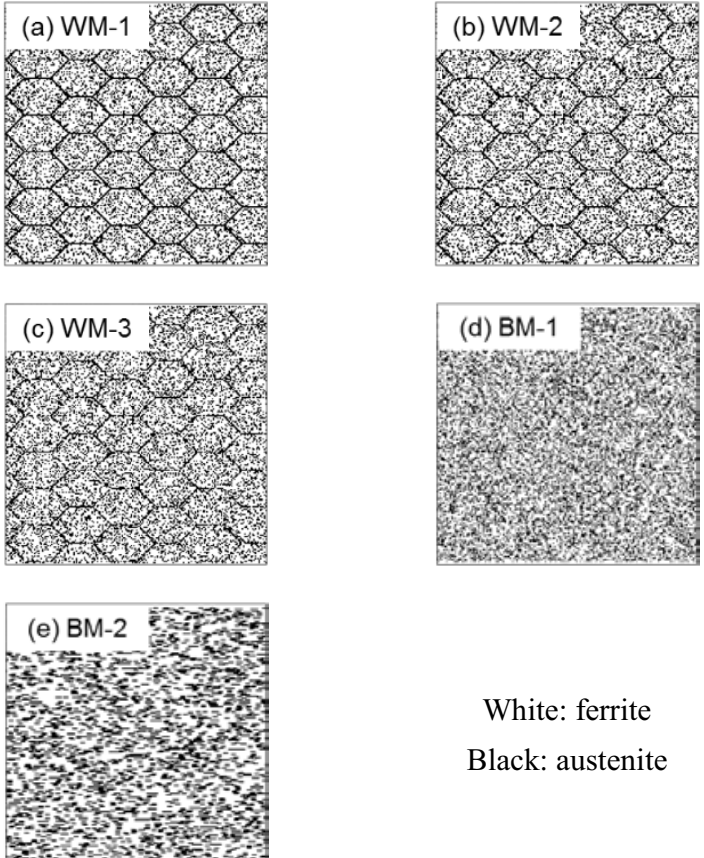

White: ferrite

Black: austenite

Fig. 3 Microstructures of duplex stainless steel and its weld used in the simulation.

\section{Result and discussion}

\subsection{Effect of microstructure in duplex stainless steel on} the hydrogen concentration

In order to investigate the effect of microstructure on hydrogen diffusion, hydrogen diffusion analysis that do not consider the effect of stress was performed first. Figure 4 shows the evolution of the averaged hydrogen concentration at the evaluation line shown in Fig. 1. Comparing the results of BM-1 and BM-2, the hydrogen diffusion rate of the latter was slow. When the aspect ratio of austenite phase increased, the hydrogen diffusion rate decreased because the austenite phase with a lower diffusion constant delayed the hydrogen diffusion. In the models WM-1, WM-2, and WM-3, when the value of the grain boundary coverage by austenite phase increased, the hydrogen diffusion rate decreased. The continuity of austenite phases affects the hydrogen diffusion behavior because it has a lower diffusion constant compared to the ferrite phase.

For each model the apparent diffusion coefficients were calculated using time-lag method ${ }^{9)}$. Table 2 shows the calculated apparent diffusion coefficients. The calculated apparent diffusion coefficient of WM-1 was much smaller than the others. This is because the grain boundary was completely covered by the austenite phase with a lower diffusion constant. In WM-2 and BM-2, there were not much difference in the apparent diffusion coefficients.
Figure 5 shows the hydrogen concentration distribution in ferrite of WM-1, WM-2, and BM-2 at 168 hours after the start of the diffusion simulation. The hydrogen concentration in ferrite phase of WM-1 was lower than the others because of the continuously distributed austenite phase prevent the diffusion on hydrogen. In WM-2 and BM-2, the averaged hydrogen concentration on the evaluation line located at $125 \mu \mathrm{m}$ above Edge-A was macroscopically the same as shown in Fig. 4, however, the microscopic hydrogen concentration was different.

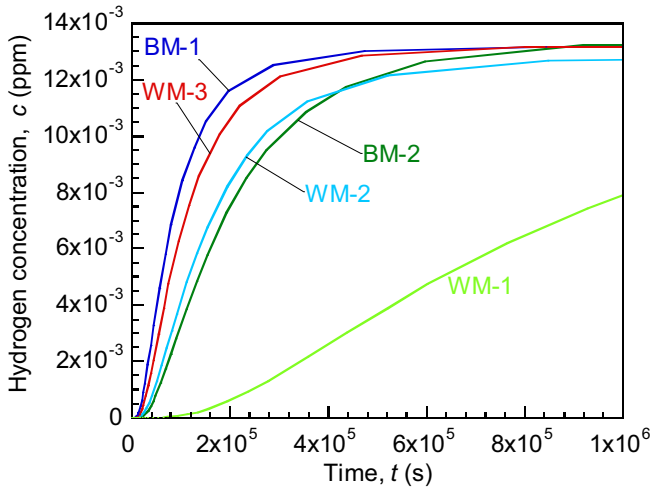

Fig. 4 Evolution of averaged hydrogen concentration.

Table 2 Apparent diffusion coefficient calculated by time-lag method.

\begin{tabular}{|c|c|}
\hline model & Apparent diffusion coefficient, $\boldsymbol{D}\left(\mathrm{m}^{2} / \mathrm{s}\right)$ \\
\hline WM-1 & $0.88 \times 10^{-14}$ \\
\hline WM-2 & $5.01 \times 10^{-14}$ \\
\hline WM-3 & $7.28 \times 10^{-14}$ \\
\hline BM-1 & $9.28 \times 10^{-14}$ \\
\hline BM-2 & $4.08 \times 10^{-14}$ \\
\hline
\end{tabular}

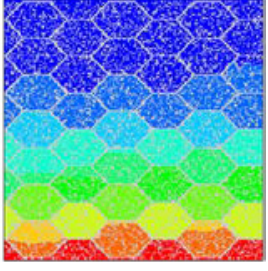

(a) WM-1

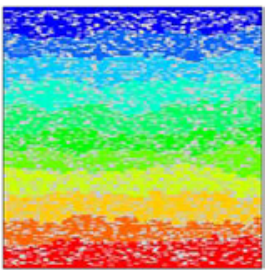

(c) $\mathrm{BM}-2$

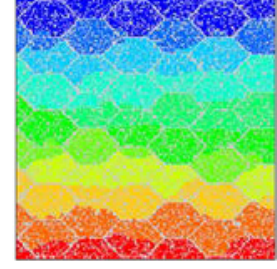

(b) WM-2

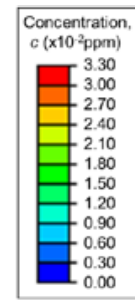

Fig. 5 Hydrogen concentration distribution in ferrite phase at 168 hours after hydrogen was applied. 
It is considered that duplex stainless steel base metal that is actually used has the microstructure as BM-2 and that duplex stainless steel weld metal that is actually used has the microstructure as WM-2 or WM-3. Therefore, when the effect of stress is not taken into account, there is no significant effect between the overall hydrogen diffusion of base metal and that of weld metal. However, the microscopic hydrogen concentration distribution is strongly influenced by austenite phase distribution. The continuity of austenite phases microscopically affects the hydrogen concentration distribution.

\subsection{Effect of microscopic stress distribution on hydrogen diffusion and distribution}

Figure 6 shows the pressure stress distribution and hydrogen concentration distribution in the ferrite grain interior of WM-1 at steady state $(3000 \mathrm{hrs})$. The diffusible hydrogen accumulated in the location where the stress concentration occurred. This is because the gradient of hydrostatic pressure is the driving force for the hydrogen diffusion, therefore, the diffusible hydrogen accumulated to the stress concentration area.
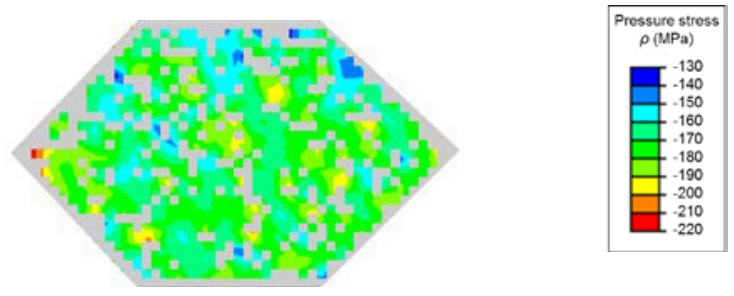

(a) Pressure stress distribution
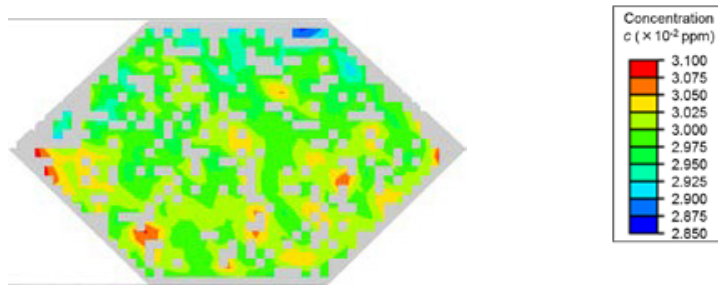

(b) Hydrogen concentration distribution

Fig. 6 Pressure stress distribution and hydrogen concentration distribution in a ferrite grain interior of WM-1 at steady state.

Figure 7 shows the transition of hydrogen concentration in ferrite phase of WM-1. The presence or absence of the microscopic stress was compared here. The hydrogen concentration distribution in the ferrite grain interiors was influenced by the microscopic stress distribution. However, the effect of the macroscopic stress distribution on the hydrogen concentration distribution was small, shown in Fig. 7. Therefore, the overall hydrogen diffusion was not affected macroscopically by the hydrostatic pressure.

Figure 8 shows the evolution of the averaged hydrogen concentration rate in WM-1 at the evaluation line shown in Fig.1. The evolution of the hydrogen concentration was not affected by the microscopic stress distribution. The microscopic stress distribution affects the local hydrogen concentration; however, it does not affect the overall diffusion behavior.

In this study, macroscopic tensile strain was uniformly applied. Therefore, although there is the pressure stress gradient in microscopic scale, the gradient is almost uniform at macroscopic scale. As a result, it is considered that hydrostatic pressure did not affect the overall diffusion behavior.

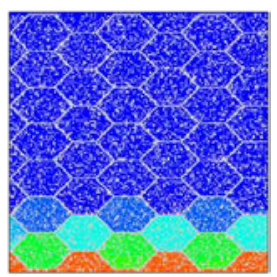

(a) $24 \mathrm{~h}$, applied stress

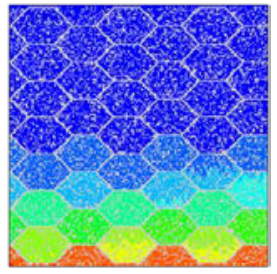

(c) $72 \mathrm{~h}$, applied stress

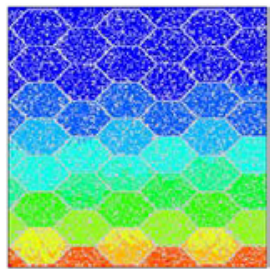

(e) $168 \mathrm{~h}$, applied stress

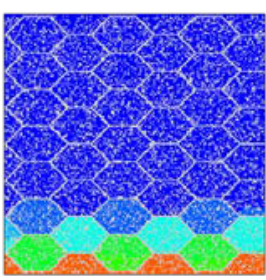

(b) $24 \mathrm{~h}$, no stress

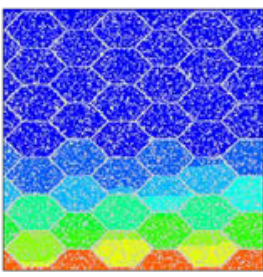

(d) $72 \mathrm{~h}$, no stress

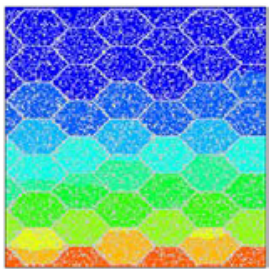

(f) $168 \mathrm{~h}$, no stress
Fig. 7 Hydrogen concentration distribution in ferrite phase of WM-1 at 24, 72 and 168 hours after hydrogen was applied.

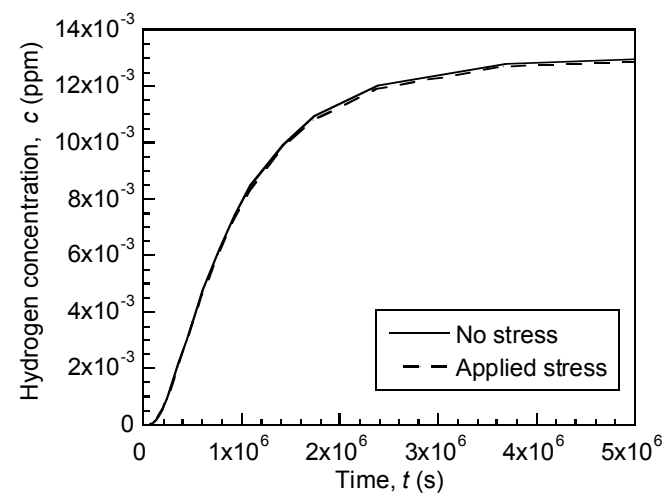

Fig. 8 Effect of microscopic stress distribution on evolution of hydrogen concentration. 


\section{Conclusions}

In this study, the effect of microstructures in duplex stainless steel on the hydrogen diffusion behavior was investigated by numerical simulations.

(1) The hydrogen diffusion behavior was strongly influenced by the continuity of austenite phases which have lower diffusion constant compared to ferrite phases.

(2) The diffusible hydrogen accumulated in the location where stress concentration occurred due to microscopically different mechanical properties

(3) The effect of stress concentration at the microscopic scale was not significant in terms of the overall diffusion behavior.

\section{Acknowledgements}

This work was partly supported by a Grant for Next Generation Researchers from the Japan Welding Engineering Society (JWES) and JSPS KAKENHI Grant Number 16K05977.

\section{Reference}

1) K. Harada: Corrosion resistant stainless steels with microduplex structure, Corrosion engineering, 26-12 (1977), 721-730.

2) V. Olden, C. Thaulow, R. Johnsen: Modelling of hydrogen diffusion and hydrogen induced cracking in supermartensitic and duplex stainless steels, Materials and design, 29-10 (2008), 1934-1948.

3) V. Olden, A. Saai, L. Jembile, R. Johnsen: FE simulation of hydrogen diffusion in duplex stainless steel, International journal of hydrogen energy, 39-2 (2014), 1156-1163.

4) K. Yokogawa, S. Fukuyama, K. Kudo: Tensile properties of ferritic-austenitic two-phase stainless steel in high pressure hydrogen at room temperature, Journal of the Japan institute of metals and materials, 48-9 (1984), 901-910.

5) F. Matsuda, H. Nakagawa, K. Shinozaki: Effect of remaining hydrogen content on cold cracking susceptibility in weld metal of high strength steels, Quarterly journal of the Japan welding society, 5-2 (1987), 244-249.

6) P. Sofronis, R. M. McMeeking: Numerical analysis of hydrogen transport near a blunting crack tip, Journal of mechanics and physics of solids, 37-3 (1989), 317-350.

7) E. Owczarek, T. Zakroczymski: Hydrogen transport in a duplex stainless steel, Acta materialia, 48-12 (2000), 3059-3070.

8) JP. Hirth: Effects of hydrogen on the properties of iron and steel, Metallurgical and materials transactions A, 11-6 (1980), 861-890.

9) T. Tsuru: Electrochemical measurements for hydrogen entry and permeation of steel, Zairyo-to-kankyo, 63-1 (2014), 3-9. 